\title{
Numerical Study of 2D Incompressible Flow in a Rectangular Domain using Chorin's Projection Method at High Reynolds Number
}

\author{
Arti Kaushik \\ Department of Mathematics \\ Maharaja Agrasen Institute of Technology, Delhi, India \\ E-mail: arti.kaushik@gmail.com
}

(Received June 2, 2018; Accepted September 2, 2018)

\begin{abstract}
In this paper the problem of a 2-D incompressible flow in a rectangular domain is analyzed. Numerical solutions of the governing equations are obtained by using Chorin's Projection method. The values of $u$-velocity, $v$-velocity and pressure are obtained numerically at different times for different positions along $x$ - axis and $y$-axis for different values of Reynolds number. The significant findings from the present analysis have been given under conclusion.
\end{abstract}

Keywords- Incompressible flow, Navier-Stokes equations, Projection method, Reynolds number.

\section{Introduction}

The area of Computational Fluid Dynamics is undergoing an extensive improvement with advances in technology. Nowadays, Navier-Stokes equations are being solved for numerous sophisticated flow problems from practical applications such as improvement of performance of modern cars and trucks, manufacturing of ceramic composite materials, air conditioning of buildings, hydrodynamic problems related to ships, submarines, etc.

The flow of a Newtonian fluid can be accurately described by Navier-Stokes equations, but an analytical solution of these equations is possible only in fully developed flows in simple geometries. In all such flows, the solution of Navier -Stokes equation is possible only by either taking many terms in the equations as zero or by just neglecting some unimportant terms in the equation. But, these simplification leads to an error in the solution. In cases where even the simplified equations cannot be solved analytically, numerical methods are employed to obtain the solution.

In compressible flows, the continuity equation is used to obtain the density and equation of state is used to obtain the pressure so as to solve the Navier-Stokes equations. But, for incompressible flows, the solution of the Navier-Stokes equations becomes more problematic because of the absence of equations which associate pressure field to the velocity field explicitly.

A number of solution techniques have been proposed by various researchers to transcend this problem. One of the technique is the Projection method which was first introduced by Chorin in which a time derivative of pressure is included in the continuity equation (Chorin, 1968). As a result the true incompressible equations need not be solved. At convergence when the time derivative becomes zero, the solution satisfies the incompressible equations.

The projection method was later applied to study the effectiveness of the coupled strongly implicit multi-grid (CSI-MG) method to determine the solutions of high-Re fine-mesh flow (Ghia 
International Journal of Mathematical, Engineering and Management Sciences

Vol. 4, No. 1, 157-169, 2019

https://dx.doi.org/10.33889/IJMEMS.2019.4.1-014

et al., 1982). A numerical method based on a fractional-step or time-splitting, scheme was developed for the solution of three-dimensional, time-dependent incompressible flows (Kim and Moin, 1985). For a time dependent viscous flow model, the pressure correction method was developed which was later improved into a second-order projection method for variable-density flows (Bell et al., 1991, Bell and Marcus, 1992). The method is applicable for both finite amplitude density variations and for fluids that are modeled using a Boussinesq approximation. The errors that generate in the discrete solutions using Chorin's method were obtained by applying asymptotic error analysis techniques (Wetton, 1996).The accuracy of projection method approximations was examined in the case of the initial-boundary-value problem for the incompressible Navier-Stokes equations and order of convergence of certain projection methods were discussed (Brown et al., 2001). Numerical simulations for a 2D lid-driven cavity flow were obtained and accurate results were presented for steady solutions as well as for periodic solutions (Bruneau and Saad, 2006). A review on the projection methods categorized these methods into three classes, namely, the pressure-correction method, the velocity-correction method and the consistent splitting method (Guermond et al, 2006). Using finite differencing, an explicit pressure-correction projection method was presented and checked on standard cases of a liddriven cavity flow, flow past a cylinder and flow over a backward facing step (Hafiz et al., 2015).

In the view of above mentioned literature, it is clear that Chorin's Projection method is mostly applied in the framework of the finite element method and it has not been studied widely for finite difference discretization. Due to this reason, we have been motivated by present investigation. Our aim in this paper is to develop a two-dimensional incompressible flow model in a rectangular domain using Chorin's Projection Method using finite difference discretization and investigate it numerically at different Reynolds numbers. The results obtained show good agreement with the previous available data.

\section{Problem Formulation}

We consider an unsteady, 2D incompressible flow in a rectangular domain. All fluid properties are assumed to be constant. We assume no body forces and no volumetric heat addition. Under these assumptions, the 2D incompressible Navier- Stokes equations (Anderson and Wendt, 1995) are given by:

Continuity: $\quad \frac{\partial u}{\partial x}+\frac{\partial v}{\partial y}=0$

$X$ momentum: $\quad \frac{\partial u}{\partial t}+\frac{\partial p}{\partial x}=-\frac{\partial\left(u^{2}\right)}{\partial x}-\frac{\partial(u v)}{\partial y}+\frac{1}{\operatorname{Re}}\left(\frac{\partial^{2} u}{\partial x^{2}}+\frac{\partial^{2} u}{\partial y^{2}}\right)$

Y momentum: $\frac{\partial v}{\partial t}+\frac{\partial p}{\partial y}=-\frac{\partial(u v)}{\partial x}-\frac{\partial\left(v^{2}\right)}{\partial y}+\frac{1}{\operatorname{Re}}\left(\frac{\partial^{2} v}{\partial x^{2}}+\frac{\partial^{2} v}{\partial y^{2}}\right)$

on the rectangular domain $\Omega=[0,1] \times[0,1]$ which is fixed in time (Figure 1). Assuming no-slip boundary conditions on each wall, the boundary conditions are: 
International Journal of Mathematical, Engineering and Management Sciences

Vol. 4, No. 1, 157-169, 2019

https://dx.doi.org/10.33889/IJMEMS.2019.4.1-014

$$
\begin{array}{ll}
u(x, 1)=u_{N} & v(x, 1)=0 \\
u(x, 0)=u_{S} & v(x, 0)=0 \\
u(0, y)=0 & v(0, y)=v_{W} \\
u(1, y)=0 & v(1, y)=v_{E} .
\end{array}
$$

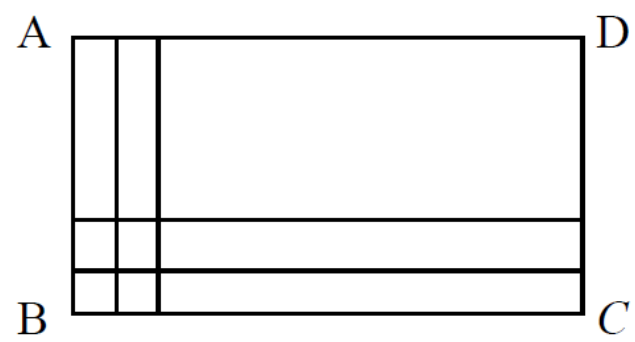

Figure 1. Rectangular domain

\section{Computational Procedure}

The governing equations of a compressible flow are different from those of incompressible flow in sense of their mathematical behavior. The governing equations for a compressible flow exhibit a hyperbolic behavior whereas the incompressible equations exhibit a mixed parabolic-elliptic behavior. To apply the compressible flow methods for the solution of incompressible flows, we need to modify the character of the equations. The difference in character is due to the fact that the continuity equation for incompressible flow does not involve a time derivative term which is present in the continuity equation for compressible flow in the form of time derivative of the density.

So, to modify the nature of the equations for incompressible flow from mixed parabolic-elliptic to hyperbolic we need to include a time derivative term to the continuity equation. This is done by taking time derivative of the pressure term. The time history thus generated cannot be accurate but, at convergence, when the time derivative becomes zero, the solution agrees with the incompressible equations. This method was developed by Chorin which provided a foundation for the development of a number of versions. The method is discussed below:

The incompressible Navier-Stokes equation may be written as

$$
\frac{\partial \mathbf{u}}{\partial t}+(\mathbf{u} \cdot \nabla) \mathbf{u}=-\nabla p+v \nabla^{2} \mathbf{u}
$$

$\nabla \cdot \mathbf{u}=0$

With the boundary condition

$$
\left.\mathbf{u}\right|_{\Omega}=\mathbf{u}_{b}
$$


International Journal of Mathematical, Engineering and Management Sciences

Vol. 4, No. 1, 157-169, 2019

https://dx.doi.org/10.33889/IJMEMS.2019.4.1-014

Where, $\boldsymbol{u}$ is the fluid velocity, $p$ is the pressure and $v$ is the kinematic viscosity of the fluid. Since, the left-hand side of Eq. (4) is Hodge decomposition, it can be written in parallel projection composition as

$$
\frac{\partial \mathbf{u}}{\partial t}=\mathbf{P}\left[-(\mathbf{u} . \nabla) \mathbf{u}+v \nabla^{2} \mathbf{u}\right]
$$

Where, $\boldsymbol{P}$ is the operator that projects a vector field onto the space of divergence-free vector fields with appropriate boundary conditions.

Using the momentum equation explicitly, we first compute an intermediate velocity, $\mathbf{u}^{i}$

$$
\begin{aligned}
& \frac{\mathbf{u}^{i}-\mathbf{u}^{n}}{\Delta t}+\nabla \tilde{p}=-[(\mathbf{u} . \nabla) \mathbf{u}]^{n+1 / 2}+\frac{v}{2} \nabla^{2}\left(\mathbf{u}^{i}+\mathbf{u}^{n}\right) \\
& B\left(\mathbf{u}^{i}\right)=0
\end{aligned}
$$

Where, $\tilde{p}$ is an approximation to $p^{n+1 / 2}$ and $B\left(\mathbf{u}^{i}\right)$ a boundary condition for $\boldsymbol{u}^{i}$ which must be specified. Now, in the projection step, we correct the intermediate velocity, $\mathbf{u}^{i}$

$$
\begin{aligned}
& \mathbf{u}^{i}=\mathbf{u}^{n+1}+\Delta t \nabla \psi^{n+1} \\
& \nabla . \mathbf{u}^{n+1}=0
\end{aligned}
$$

Using boundary conditions consistent with $B\left(\mathbf{u}^{i}\right)=0$ and $\left.\mathbf{u}^{n+1}\right|_{\Omega}=\mathbf{u}_{b}{ }^{n+1}$.

Updating the pressure $p^{n+1 / 2}=\tilde{p}+L\left(\psi^{n+1}\right)$, where, the function $L$ shows that of $p^{n+1 / 2}$ depends on $\psi^{n+1}$.After the completion of a given time step, the predicted velocity $\boldsymbol{u}^{i}$ is ignored and is not used again at that or later time steps.

\section{Numerical Computation}

In order to obtain the unknown variables $u$-velocity, $v$-velocity and pressure $p$, numerical computations are carried out at different time intervals and for different values of $\operatorname{Re}=500,1000$, 2000 and displayed. While doing the computations, we have taken $0 \leq x \leq 1 ; 0 \leq y \leq 1 ; \Delta x=$ $0.01 ; \Delta y=0.01 ; \Delta t=0.01$. The computations are done by following Chorin's Projection method which has been described under Section 3. The same algorithm has been implemented in MATLAB programming language. The unknown quantities are obtained at different node points and at different time intervals for different values of Re.

The computed values of unknown quantities $u$-velocity, $v$-velocity and pressure at different nodes and different values of $\operatorname{Re}$ at $t=15$ are given under Tables 1 to 3 . 
International Journal of Mathematical, Engineering and Management Sciences

Vol. 4, No. 1, 157-169, 2019

https://dx.doi.org/10.33889/IJMEMS.2019.4.1-014

Table 1. $u$-velocity at different nodes and different values of Re at $t=15$

\begin{tabular}{|c|c|c|c|c|}
\hline$x$ coordinate & $y$ coordinate & $u$ velocity at $\mathrm{Re}=500$ & $u$ velocity at $\mathrm{Re}=1000$ & $u$ velocity at $\mathrm{Re}=2000$ \\
\hline 0.1 & 0.1 & -0.0136 & -0.0140 & -0.0136 \\
\hline 0.2 & 0.2 & -0.1010 & -0.1121 & -0.0992 \\
\hline 0.3 & 0.3 & -0.1886 & -0.1739 & -0.1398 \\
\hline 0.4 & 0.4 & -0.1614 & -0.1079 & -0.0789 \\
\hline 0.5 & 0.5 & -0.0555 & -0.0244 & 0.0035 \\
\hline 0.6 & 0.6 & 0.0337 & 0.0550 & 0.0788 \\
\hline 0.7 & 0.7 & 0.0743 & 0.0820 & 0.0818 \\
\hline 0.8 & 0.8 & 0.1160 & 0.0881 & 0.1314 \\
\hline
\end{tabular}

Table 2. $v$-velocity at different nodes and different values of Re at $t=15$

\begin{tabular}{|c|c|c|c|c|}
\hline$x$ coordinate & $y$ coordinate & $v$ velocity at $\mathrm{Re}=500$ & $v$ velocity at $\mathrm{Re}=1000$ & $v$-velocity at $\mathrm{Re}=2000$ \\
\hline 0.1 & 0.1 & 0.0114 & 0.0108 & 0.0094 \\
\hline 0.2 & 0.2 & 0.0826 & 0.0991 & 0.0937 \\
\hline 0.3 & 0.3 & 0.1565 & 0.0991 & 0.1671 \\
\hline 0.4 & 0.4 & 0.1264 & 0.1076 & 0.1036 \\
\hline 0.5 & 0.5 & 0.0156 & 0.0080 & 0.0117 \\
\hline 0.6 & 0.6 & -0.0741 & -0.0776 & -0.0759 \\
\hline 0.7 & 0.7 & -0.1149 & -0.0989 & -0.0807 \\
\hline 0.8 & 0.8 & -0.2128 & -0.1369 & -0.0604 \\
\hline
\end{tabular}

Table 3. Pressure at different nodes and different values of Re at $t=15$

\begin{tabular}{|c|c|c|c|c|}
\hline$x$ coordinate & $y$ coordinate & Pressure at Re $=500$ & Pressure at Re $=1000$ & Pressure at Re $=2000$ \\
\hline 0.1 & 0.1 & $-4.2264 \mathrm{e}-007$ & $-2.5332 \mathrm{e}-007$ & $-2.0477 \mathrm{e}-007$ \\
\hline 0.2 & 0.2 & $-2.3526 \mathrm{e}-005$ & $-2.7590 \mathrm{e}-005$ & $-2.1190 \mathrm{e}-005$ \\
\hline 0.3 & 0.3 & $-2.0342 \mathrm{e}-004$ & $-2.3961 \mathrm{e}-004$ & $-1.9647 \mathrm{e}-004$ \\
\hline 0.4 & 0.4 & $-5.7767 \mathrm{e}-004$ & $-5.4279 \mathrm{e}-004$ & $-4.1608 \mathrm{e}-004$ \\
\hline 0.5 & 0.5 & $-8.1592 \mathrm{e}-004$ & $-6.7301 \mathrm{e}-004$ & $-5.1157 \mathrm{e}-004$ \\
\hline 0.6 & 0.6 & $-7.7936 \mathrm{e}-004$ & $-6.0293 \mathrm{e}-004$ & $-4.0819 \mathrm{e}-004$ \\
\hline 0.7 & 0.7 & $-5.3602 \mathrm{e}-004$ & $-3.7465 \mathrm{e}-004$ & $-2.1279 \mathrm{e}-004$ \\
\hline 0.8 & 0.8 & $2.1488 \mathrm{e}-004$ & $8.9427 \mathrm{e}-006$ & $-6.1256 \mathrm{e}-005$ \\
\hline
\end{tabular}

The numerical values of $u$-velocity of the fluid at time $t=15$ for different values of $\operatorname{Re}$ are illustrated in Figure 2. 
International Journal of Mathematical, Engineering and Management Sciences

Vol. 4, No. 1, 157-169, 2019

https://dx.doi.org/10.33889/IJMEMS.2019.4.1-014
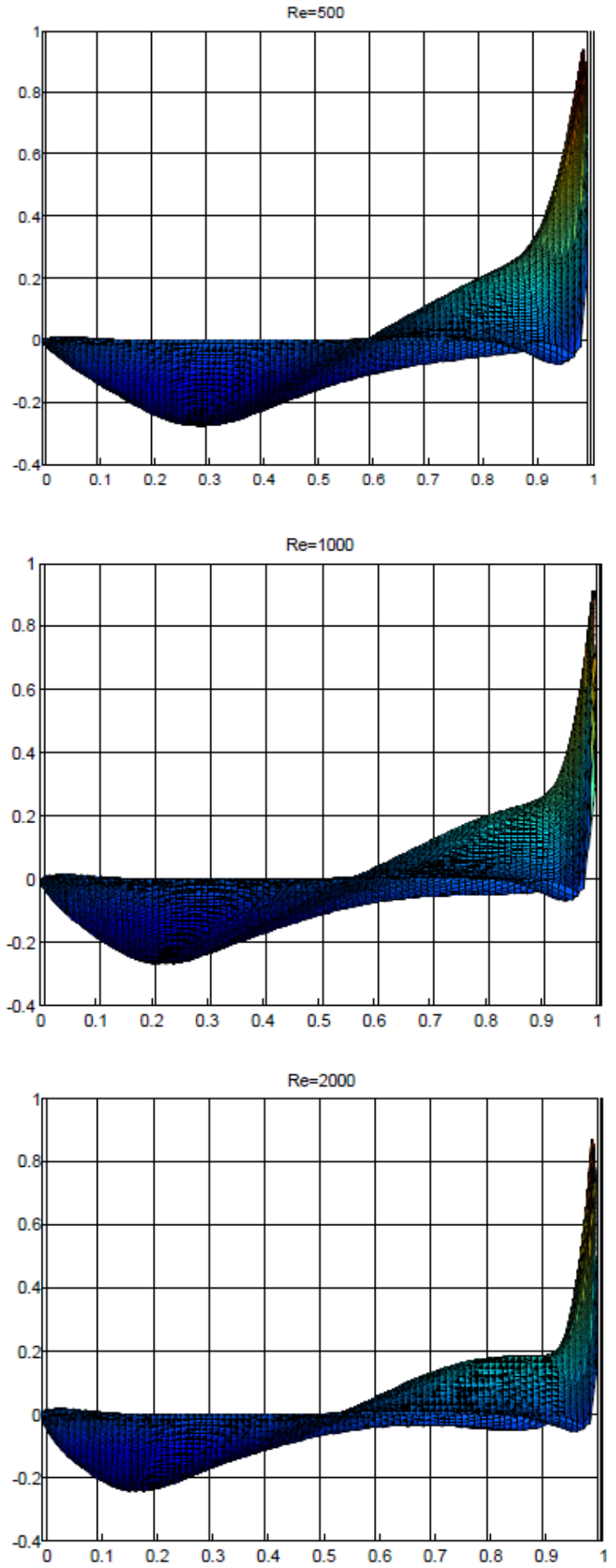

Figure 2. $u$-velocity at $\mathrm{Re}=500, \mathrm{Re}=1000$ and $\mathrm{Re}=2000$ 
International Journal of Mathematical, Engineering and Management Sciences

Vol. 4, No. 1, 157-169, 2019

https://dx.doi.org/10.33889/IJMEMS.2019.4.1-014

The numerical values of $v$-velocity of the fluid at time $t=15 \mathrm{sec}$ for different values of Re are illustrated in Figure 3.
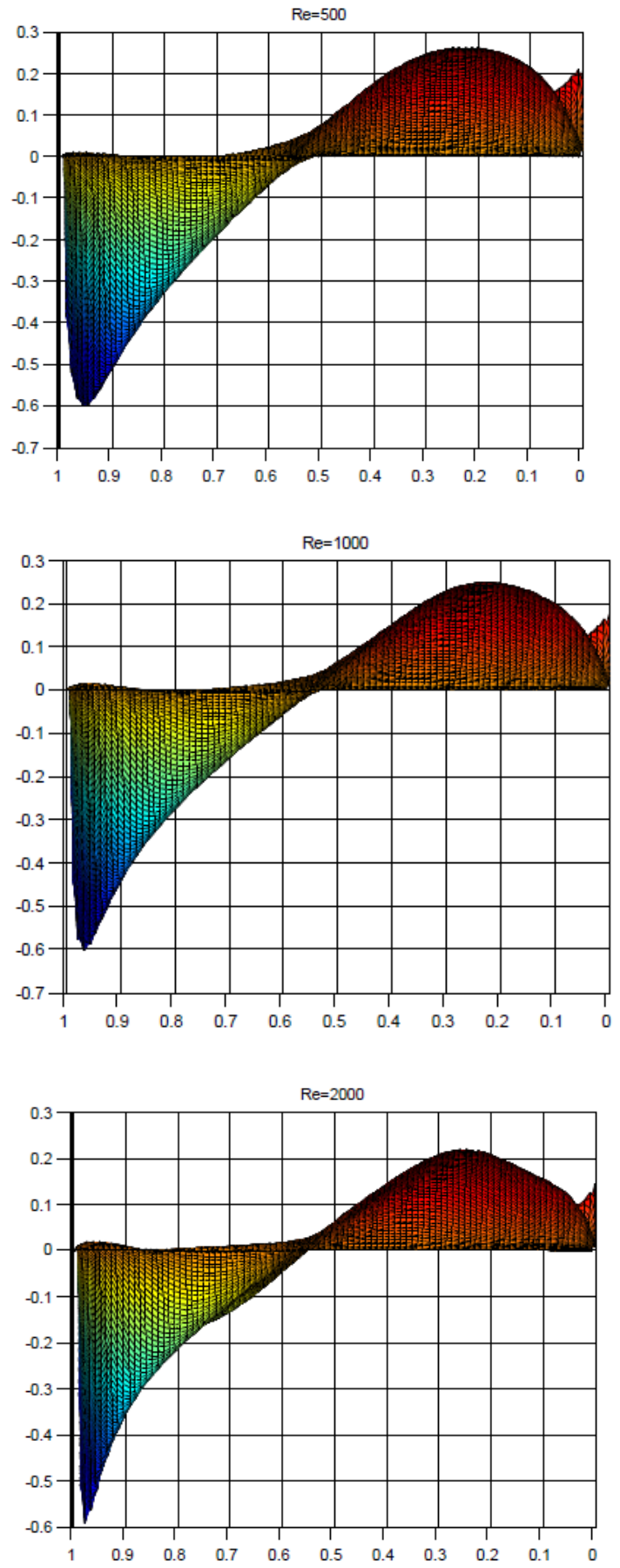

Figure 3. $v$-velocity at $\mathrm{Re}=500, \mathrm{Re}=1000$ and $\mathrm{Re}=2000$ 
International Journal of Mathematical, Engineering and Management Sciences

Vol. 4, No. 1, 157-169, 2019

https://dx.doi.org/10.33889/IJMEMS.2019.4.1-014

The variation of $u$-velocity with $y$ and variation of $v$-velocity with $x$ are shown in Figure 4 .
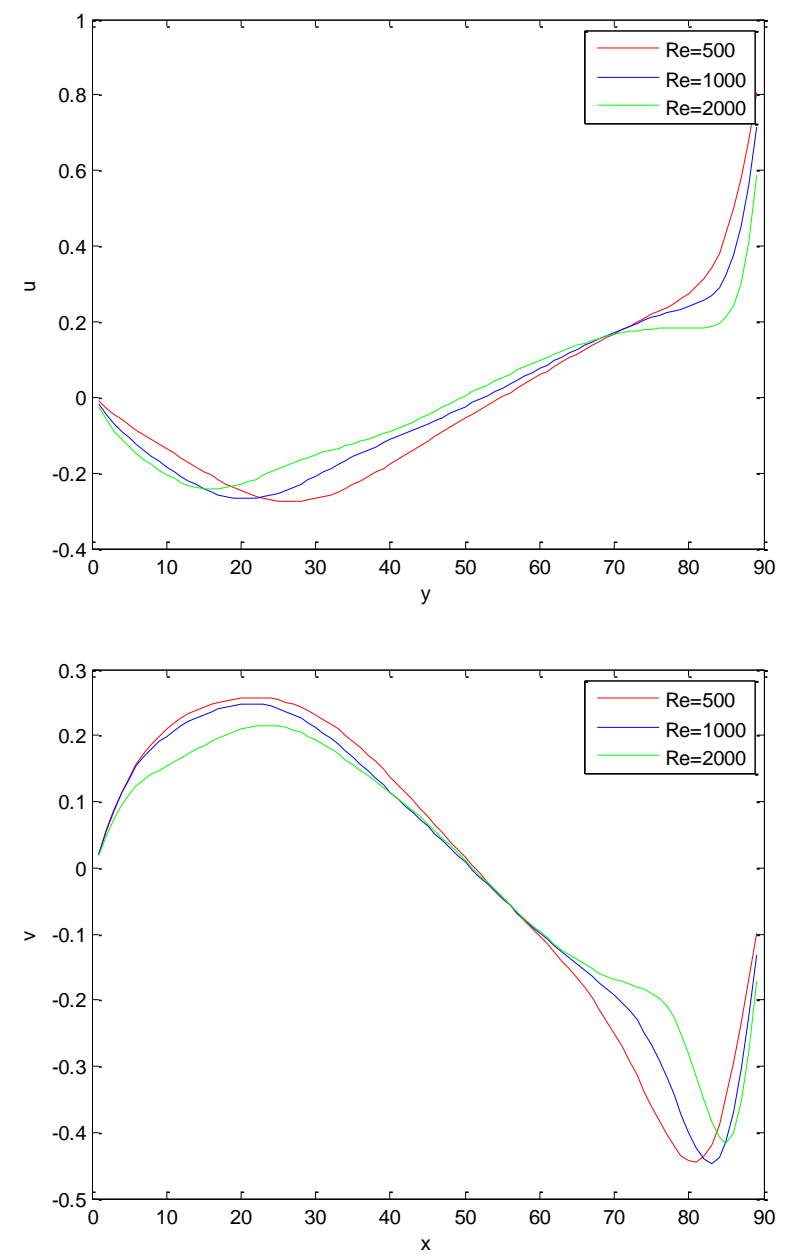

Figure 4. Variation of $u$-velocity and $v$-velocity at $\mathrm{Re}=500, \mathrm{Re}=1000$ and $\mathrm{Re}=2000$

The numerical solutions for the pressure at times $t=5 \mathrm{sec}$ and $15 \mathrm{sec}$ at all nodes in the domain for different values of Re have been computed and are illustrated in Figures. 5 and 6. 
International Journal of Mathematical, Engineering and Management Sciences Vol. 4, No. 1, 157-169, 2019

https://dx.doi.org/10.33889/IJMEMS.2019.4.1-014
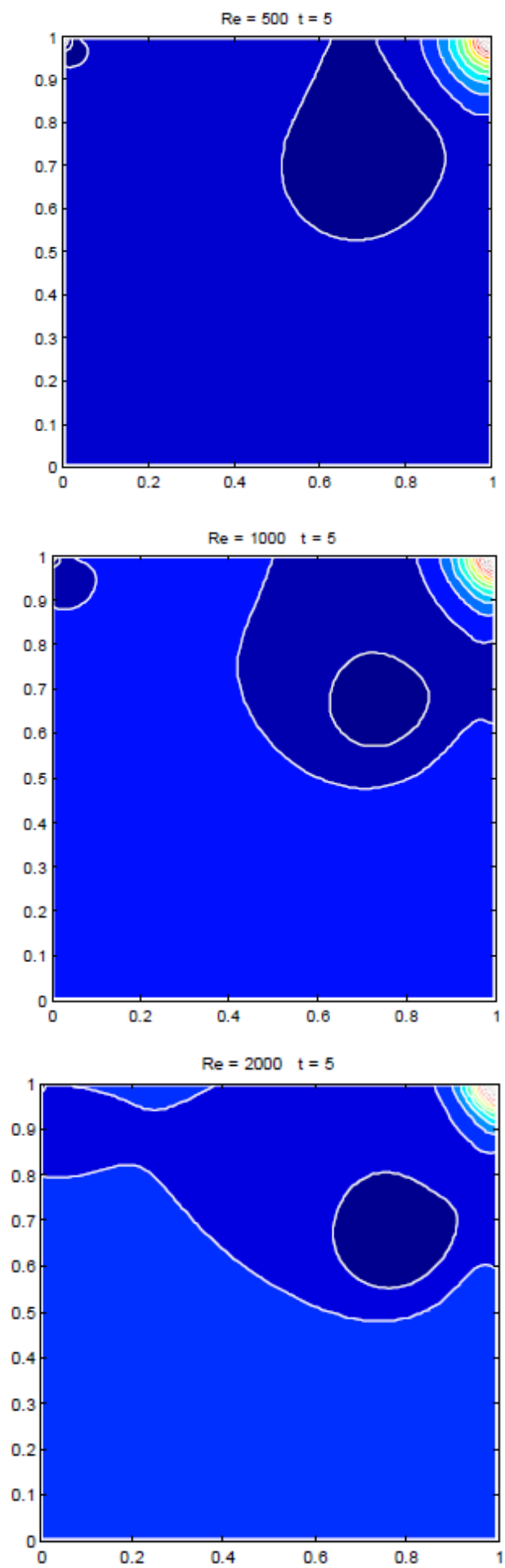

Figure 5. Pressure at $t=5 \mathrm{sec}$ for different values of $\mathrm{Re}$ 
International Journal of Mathematical, Engineering and Management Sciences Vol. 4, No. 1, 157-169, 2019

https://dx.doi.org/10.33889/IJMEMS.2019.4.1-014
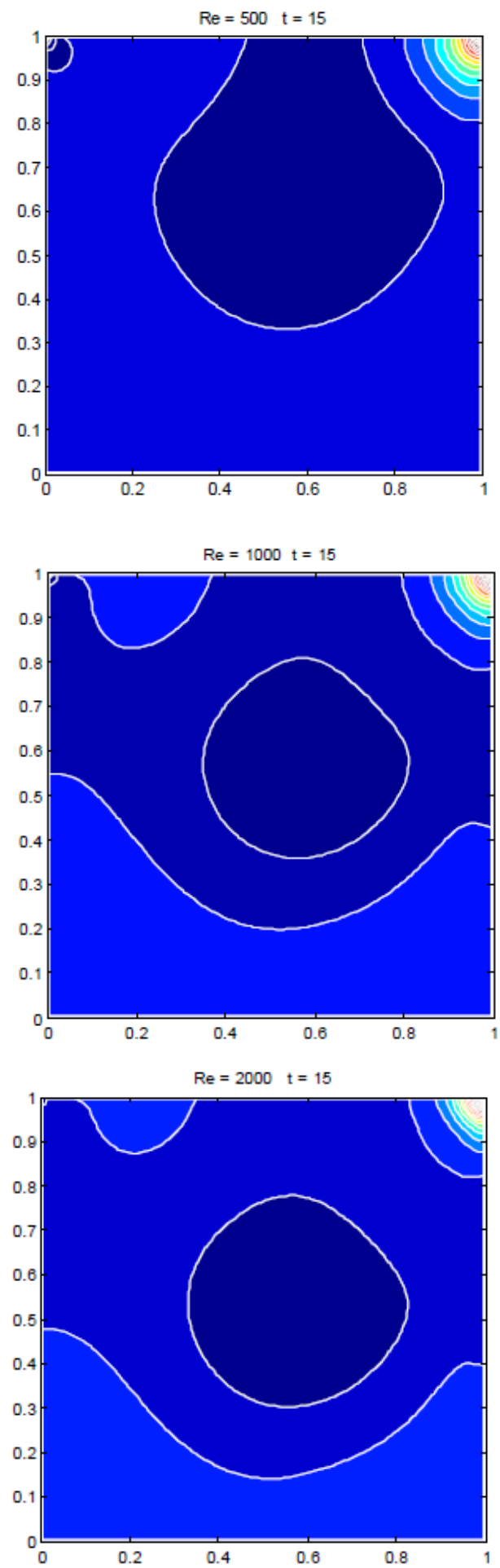

Figure 6. Pressure at $t=15 \mathrm{sec}$ for different values of Re 
International Journal of Mathematical, Engineering and Management Sciences

Vol. 4, No. 1, 157-169, 2019

https://dx.doi.org/10.33889/IJMEMS.2019.4.1-014

The streamlines for the incompressible flow at time $t=15 \mathrm{sec}$ for different values of $\operatorname{Re}$ are illustrated in Figure 7.
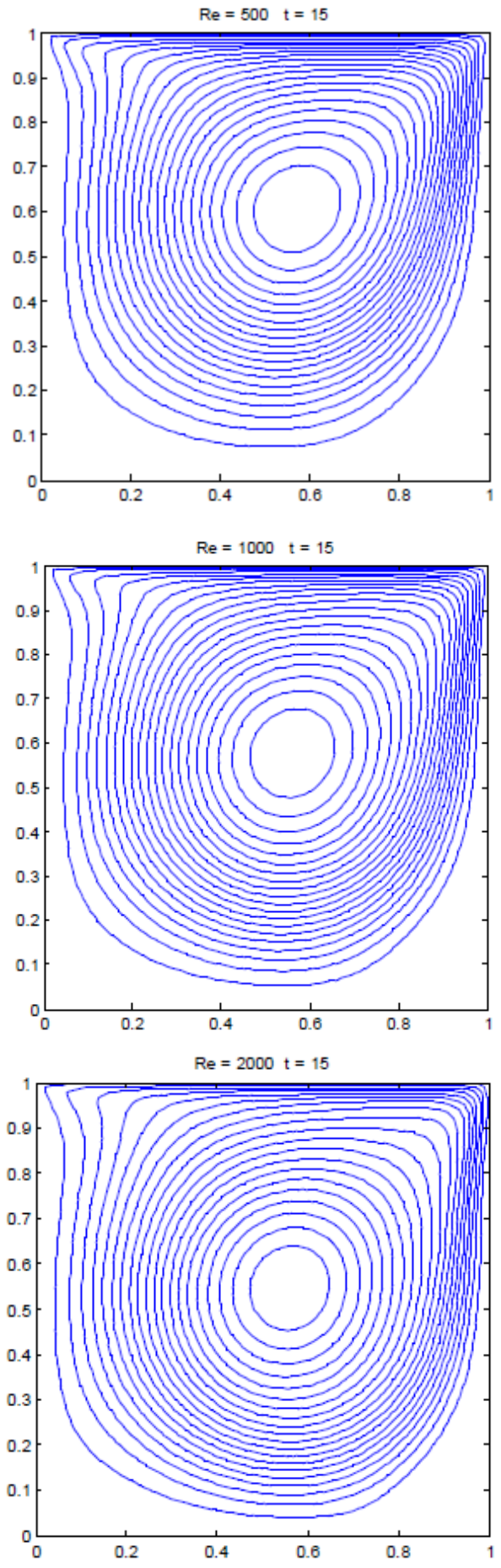

Figure 7. Streamlines for the incompressible flow at $t=15 \mathrm{sec}$ for different values of Re 
International Journal of Mathematical, Engineering and Management Sciences

Vol. 4, No. 1, 157-169, 2019

https://dx.doi.org/10.33889/IJMEMS.2019.4.1-014

\title{
5. Result and Discussion
}

(i) From Figure 2 for $u$-velocity, it is observed that the negative values of $u$-velocity decreases in magnitude with increase in values of Re. It can be seen that the as Re increases, a thin boundary layer is developing along the walls. However, the peaked value of $u$-velocity decreases as value of Re increases.

(ii) From Figure 3 for $v$-velocity, it is observed that as in case of $u$-velocity the negative values of $v$-velocity also decreases in magnitude with increase in values of Re. It can be seen that the boundary layer is thicker for lower values of Re.

(iii) Figure 4 depicts the variation of $u$-velocity with y and variation of $v$-velocity with $\mathrm{x}$. These variations are comparable to those given in Figure 8 (Hafiz et al., 2015).

(iv) From the Figure 5 and Figure 6 for the pressure at different times and different values of $\mathrm{Re}$, it has been observed that the size of the low pressure zone decreases with increase in Re. With increase in $\mathrm{Re}$, the location of low pressure zone shifts towards centre of the domain. Also, the low pressure zone at the left upper corner decreases in size with increase in $\mathrm{Re}$ and for $\mathrm{Re}=2000$ it disappears completely. The result is in agreement with that given in Figure 2 (Bruneau and Saad, 2006), where the pressure variation is given for $\operatorname{Re}=1000$.

(v) From the Figure 7 for the streamlines for different values of Re, it can be seen that the major vortex moves towards the centre of the domain with increasing value of Re, whereas the corner vortices grow in size with increase in Re. Also the vortex in left corner is bigger and stronger than the vortex in right corner of the domain. Similar results are given in Figure 4 (b) and (c) (Kim and Moin 1985), where streamlines are shown for $\mathrm{Re}=400$ and $\mathrm{Re}=2000$.

\section{Conclusion}

In this work, numerical solutions were obtained at different Reynolds numbers for a 2-D incompressible flow in a rectangular domain using Chorin's Projection Method. It was found that the size and location of low pressure zone and hence of primary vortex depends on the value of Reynolds number. It was noted that the low pressure zone shifts towards of the centre of the domain as the value of $\mathrm{Re}$ increases. The results obtained are in good agreement with the previous data available.

\author{
Nomenclature \\ $u$ - u velocity \\ $v$ - v velocity \\ $x$, $y$-Cartesian coordinates \\ $t$ - time \\ $p$-pressure \\ Re- Reynolds number
}


International Journal of Mathematical, Engineering and Management Sciences

Vol. 4, No. 1, 157-169, 2019

https://dx.doi.org/10.33889/IJMEMS.2019.4.1-014

\section{Conflict of Interest}

The author confirms that this article contents have no conflict of interest.

\section{Acknowledgement}

The author would like to express her sincere thanks to the referees and for their valuable suggestions towards to the improvement of the paper.

\section{References}

Anderson, J. D., \& Wendt, J. (1995). Computational fluid dynamics (Vol. 206). New York: McGraw-Hill.

Bell, J. B., \& Marcus, D. L. (1992). A second-order projection method for variable-density flows. Journal of Computational Physics, 101(2), 334-348.

Bell, J., Howell, L., \& Colella, P. (1991, April). An efficient second-order projection method for viscous incompressible flow. In 10th Computational Fluid Dynamics Conference (p. 1560).

Brown, D. L., Cortez, R. \& Minion, M. L.(2001). Accurate projection methods for the incompressible Navier-Stokes equations. Journal of Computational Physics, 168(2), 464-49.

Bruneau, C. H., \& Saad, M. (2006). The 2D lid-driven cavity problem revisited. Computers \& Fluids, $35(3), 326-348$.

Chorin, A. J. (1968). Numerical solution of the Navier-Stokes equations. Mathematics of Computation, 22(104), 745-762.

Ghia, U., Ghia, K. N., \& Shin, C. T. (1982). High-Re solutions for incompressible flow using the NavierStokes equations and a multigrid method. Journal of Computational Physics, 48(3), 387-411.

Guermond, J. L., Minev, P., \& Shen, J. (2006). An overview of projection methods for incompressible flows. Computer Methods in Applied Mechanics and Engineering, 195(44-47), 6011-6045.

Hafiz, U. A., Hoda, A., \& Asrar, W. (2015). A numerical investigation of explicit pressure-correction projection methods for incompressible flows. Engineering Applications of Computational Fluid Mechanics, 9(1), 50-65.

Kim, J., \& Moin, P. (1985). Application of a fractional-step method to incompressible Navier-Stokes equations. Journal of Computational Physics, 59(2), 308-323.

Wetton, B. R. (1996). Error analysis for Chorin's original fully discrete projection method and regularizations in space and time. SIAM Journal of Numerical Analysis, 34(5), 1683-1697. 